tos intelectual, académico y científico, sino también aquellas personas más o menos informadas que sientan curiosidad e interés por el tema de alguno de estos diálogos, contribuirán a definir preguntas y a buscar respuestas sobre los temas abordados. Constituyen una pieza clave del acontecimiento, en tanto que recogen la dualidad entre la razón y la experimentación combinando la palabra con las más diversas formas de expresión artística.

Entre los 45 diálogos organizados, el denominado "Derechos culturales y desarrollo humano. Nuevos espacios multiculturales" constituye un punto de referencia para explorar el nuevo papel de la cultura en la sociedad del siglo XXI. Este espacio -dirigido, hasta su fallecimiento el pasado 7 de febrero, por Eduard Delgado y la Fundación Interarts, en colaboración con la UNESCO y la Agencia Española de Cooperación Internacional (AECI)- está previsto como un gran encuentro de expertos, organizaciones internacionales y redes de distintos campos relacionados con los derechos culturales, los derechos humanos, la diversidad cultural, las políticas y el desarrollo humano. Poniendo en relación a todos estos agentes de diversos campos y países, el evento pretende tener un impacto duradero en los debates que existen en la actualidad sobre derechos culturales e indicadores culturales del desarrollo. El foro de debate y reflexión tendrá lugar en agosto y se plantea estas líneas de trabajo:

$>$ Creación de nuevas redes y sistemas de trabajo, nueva agenda para las redes de cultura y desarrollo y una plataforma de sociedad civil mundial para la cultura

$>$ Reformulación del derecho a participar en la vida cultural

$>$ Indicadores culturales del desarrollo humano

> Presentación de una sintesis de investigacio- nes y debates técnicos y políticos sobre derechos e indicadores

$>$ Presentación de diferentes experiencias territoriales de diferentes regiones del mundo

El Diálogo es sólo una parte del proceso mucho más vasto que la Fundación Interarts está preparando en estrecha colaboración con agencias y expertos internacionales. Dicho proceso comprende una serie de reuniones regionales con expertos y actores de la sociedad civil en distintas partes del mundo, la recopilación de material documental así como de ejemplos de buenas prácticas, y la investigación sobre las percepciones regionales de los derechos culturales.

Diálogo sobre derechos culturales y desarrollo
humano
Fórum Universal de las Culturas, Barcelona 2004
23 al 27 de agosto de 2004
Centre de Convencions Internacional de Barcelona
(CCIB)
Más información:
Fundación Interarts
Barcelona
Tel.: 934877022
Fax: 934872644
Web: www.interarts.net
Correo-e.: broldan@interarts.net

Razones para estar en contra del Fórum

Asambleas de resistencias al Fórum 2004

Web: www.moviments.net/resistencies2004

Correo-e.: resistencias2004@moviments.net

Indymedia Barcelona - Barcelona Independent Media Center

Web: barcelona.indymedia.org/index.php

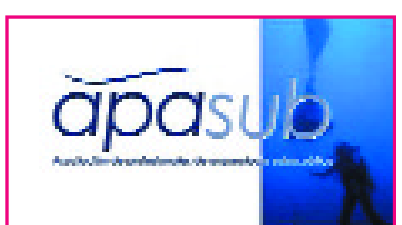

Página principal de la web de la asociación: www. arqueologiasubacuatica.org

\title{
Nace APASub, asociación de profesionales de la arqueología subacuática
}

La Asociación de Profesionales en Arqueología Subacuática, que ahora nace con una vocación de servicio para la comunicación entre los técnicos que investigan, administran, gestionan y trabajan sobre el patrimonio cultural subacuático, se crea con el fin de investigar, salvaguardar, promover y difundir el patrimonio cultural subacuático. Concienciados de la situación actual, un grupo de técnicos y estudiantes, de diferentes disciplinas (relacionadas con la arqueología subacuática) y representativos de las distintas comunidades autónomas, han desarrollado este canal de comunicación que se plasma en una Asociación de Profesionales cuyas líneas de trabajo y objetivos se han redactado de acuerdo con los principios actuales sobre protección del patrimonio cultural subacuático.
Basándonos en la Convención sobre la Protección del Patrimonio Cultural Subacuático (UNESCO. Paris, 2 de noviembre de 2001), celebrada en la Conferencia General de Naciones Unidas para la Educación, la Ciencia y la Cultura, en su $31^{\text {a }}$ reunión (véase PH38, marzo de 2002), así como en la legislación vigente de protección y actuación sobre el patrimonio cultural subacuático, APASub ha desarrollado sus estatutos, normativa interna y objetivos que conforman la línea de actuación de dicha asociación de profesionales. En el proceso de redacción de dichos documentos han colaborado y colaboran técnicos de diferentes áreas que han permitido crear los cauces y herramientas necesarias para que en un futuro, con el consenso de los demás especialistas, se convierta en nexo de unión 


\section{$020-021$}

\section{Noticias y comentarios}

\section{PH48 - Abril 2004}

y punto de referencia para los profesionales de nuestro país.

En sintesis APASub se configura como:

> Asociación de profesionales en torno a la Arqueología Subacuática.

> Alternativa no gubernamental sin ánimo de lucro. > Soporte y coordinación entre técnicos de distintas comunidades autónomas, para la difusión e intercambio de información.

> Difusión y protección del patrimonio subacuático. $>$ Coordinación de actividades en beneficio de la Arqueología Subacuática.

> Organización de eventos para la difusión del patrimonio subacuático.

Confiamos en que esta Asociación agrupe a los técnicos que actualmente gestionan el patrimonio y a los jóvenes que lo harán el día de mañana, así como que renueve las ilusiones a los profesionales que nos precedieron, y que, de ahora en adelante, podamos contar con su experiencia para una efectiva investigación, protección y difusión del patrimonio cultural subacuático.

Más información:

APASub

Web: www.arqueologiasubacuatica.org

Correo-e.: administracion@apasub.org

Carlos Cabrera Tejedor

Presidente de APASub

\section{La asociación OIKOS publica el primer número de Cuadernos de Economía de la Cultura}

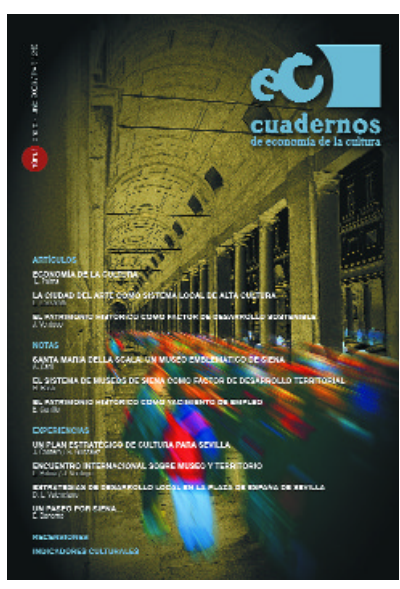

Formada por un grupo de profesionales de amplio espectro disciplinar que comparten y desean promover un foro abierto a la expresión de las ideas, la participación y la creatividad como contribución independiente al progreso de Andalucia, la asociación OIKOS se concibe como un observatorio andaluz enfocado al análisis de tendencias e instrumento de conocimiento relacionado con los aspectos culturales desde un punto de vista económico.

Uno de los fines primordiales de la asociación, recogido en sus estatutos, es "difundir a través de publicaciones en sus distintos formatos investigaciones y opinión sobre el mundo de la cultura y su relación con el desarrollo económico". En esta línea, OIKOS acaba de editar el número 1 de la revista Cuadernos de Economía de la Cultura (enerojunio 2003), una publicación que se establece como medio fundamental en el que exponer a la discusión pública los trabajos que desde la asociación y su entorno abordan un mejor conocimiento de la disciplina Economía de la Cultura. Además, la revista está abierta a la incorporación de contribuciones ajenas a la asociación y que encajen en los objetivos de la misma y en el perfil editorial de la revista.

Entendemos que una buena gestión de la cultura puede y debe producir beneficios económicos. Además, el uso de herramientas del análisis económico puede ayudarnos a entender mejor las claves de funcionamiento de un sector que, tanto desde lo privado como desde lo público, se erige en una pieza clave del Estado del Bienestar. La cultura, efectivamente, ha de concebirse como un factor de desarrollo pero también de cohesión social. Estos objetivos deben conseguirse mediante un proceso de democratización cultural que pretendemos estimular desde la revista.
La revista Cuadernos de Economía de la Cultura viene a llenar un vacío entre las publicaciones periódicas de ámbito nacional. Al mismo tiempo, desea servir de vehículo para la comparación, el contraste y la cooperación que fortalezcan vínculos regionales y transnacionales. La información contenida en sus páginas pretende ejercer como instrumento para la toma de decisiones de los gestores culturales, las instancias públicas y los investigadores.

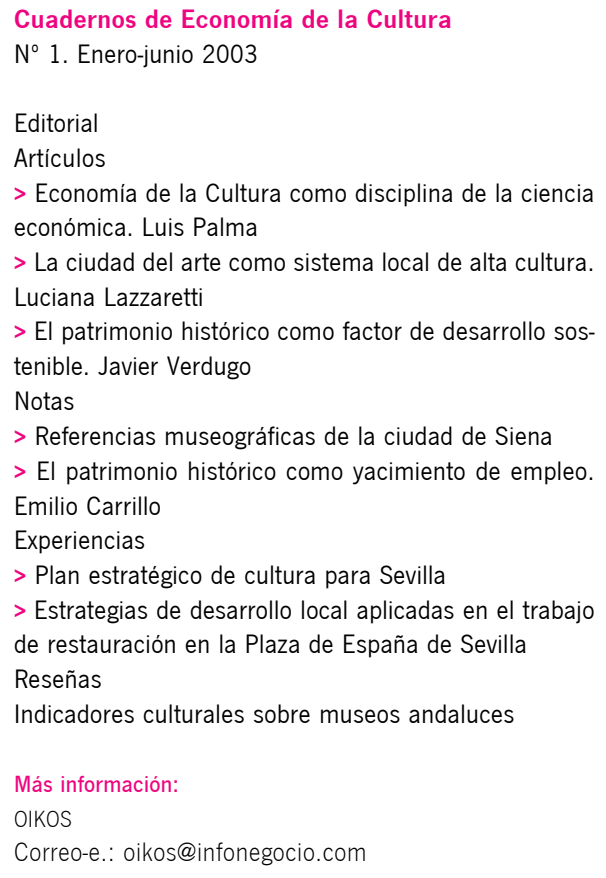

$>$ Economía de la Cultura como disciplina de la ciencia económica. Luis Palma

$>$ La ciudad del arte como sistema local de alta cultura. Luciana Lazzaretti

$>$ El patrimonio histórico como factor de desarrollo sostenible. Javier Verdugo

Notas

$>$ Referencias museográficas de la ciudad de Siena

$>$ El patrimonio histórico como yacimiento de empleo. Emilio Carrillo

Experiencias

$>$ Plan estratégico de cultura para Sevilla

$>$ Estrategias de desarrollo local aplicadas en el trabajo de restauración en la Plaza de España de Sevilla Reseñas

Indicadores culturales sobre museos andaluces

Más información

OIKOS

Correo-e.: oikos@infonegocio.com

\section{José María Medianero Hernández}

Luis Palma Martos

Miembros de OIKOS

Universidad de Sevilla 